\title{
Empirical Adequacy and Ramsification
}

\author{
Jeffrey Ketland
}

\begin{abstract}
Structural realism has been proposed as an epistemological position interpolating between realism and sceptical anti-realism about scientific theories. The structural realist who accepts a scientific theory $\Theta$ thinks that $\Theta$ is empirically correct, and furthermore is a realist about the 'structural content' of $\Theta$. But what exactly is 'structural content'? One proposal is that the 'structural content' of a scientific theory may be associated with its Ramsey sentence $\mathfrak{R}(\Theta)$. However, Demopoulos and Friedman argued, using ideas drawn from Newman's earlier criticism of Russell's structuralism, that this move fails to achieve an interesting intermediate position between realism and anti-realism. Rather, $\mathfrak{R}(\Theta)$ adds little content beyond the instrumentalistically acceptable claim that the theory $\Theta$ is empirically adequate. Here, we formulate carefully the crucial claim of Demopoulos and Friedman, and show that the Ramsey sentence $\mathfrak{R}(\Theta)$ is true just in case $\Theta$ possesses a full model which is empirically correct and satisfies a certain cardinality condition on its theoretical domain. This suggests that structural realism is not a position significantly different from the anti-realism it attempts to distinguish itself from.
\end{abstract}

\section{Introduction}

2. Technical Framework

3. Ramsification

4. Empirical Adequacy

5. Ramsification $\approx$ Empirical Adequacy + Cardinality Constraint

6. Conclusion

\section{Introduction}

Frank Ramsey ([1929]) proposed a method of formalizing our scientific theories of the world as follows. The Ramsey sentence $\mathfrak{R}(\Theta)$ of a given (finitely axiomatized) theory $\Theta$ is obtained by eliminating theoretical predicates, replacing them uniformly with predicate (i.e., second-order) variables and prefixing the result with existential second-order quantifiers. ${ }^{1}$ The epistemological significance of this technical construction it that it perhaps offers the possibility of defending a plausible intermediary or 'third way' position between realism and anti-realism (under whatever guise: positivism, empiricism or instrumentalism). This position has come to be termed 'structural realism' (see Worrall [1989] and Zahar [2001]).

One canonical version of sceptical anti-realism about theoretical science is constructive empiricism (van Fraassen [1980]). This anti-realist position holds that the aim of science is to construct empirically adequate theories and the maximal

\footnotetext{
${ }^{1}$ Carnap ([1966], Chapter 26 'The Ramsey Sentence') contains a detailed discussion of ramsification and its philosophical significance.
} 
epistemic attitude to take towards a well-corroborated, explanatory, predictively successful, etc., theory is to believe that the theory is empirically adequate. This is weaker than the realist's commitment to the approximate truth of the suitably certified theory - the theory conforms to reality, at a certain level of approximation. ${ }^{2}$ Attempting to delineate the structural realist's middle way, Worrall ([1989]) proposed that, at least in certain cases, we can defend a commitment to more than the empirical adequacy of the theory in question, but less than the approximate truth of the theory. We can sometimes defend a commitment to a theory's empirical adequacy plus its 'structural content', an aspect of transempirical content which is preserved under even radical theory change. However, the required notion of 'structural content' of a scientific theory is notoriously unclear. One proposal, endorsed by Worrall and Zahar ([2001]), is that the structural content of a theory $\Theta$ is given by its Ramsey sentence $\mathfrak{R}(\Theta)$. More exactly, the structural content of a theory $\Theta$ is what $\mathfrak{R}(\Theta)$ 'adds' beyond the empirical adequacy claim for $\Theta$.

Having briefly sketched the epistemological background, we now face the following problem. Given a theory $\Theta$, can we clarify the relationship between the claim that $\mathfrak{R}(\Theta)$ is true and the claim that $\Theta$ is empirically adequate? If I am correct, there is an intimate relation between these claims. We show that, at least on the conventional understanding of second-order logic, the adoption of 'ramsification' as a correct representation of structural content fails to achieve the requisite intermediate position between realism and sceptical anti-realism. Rather, as Demopoulos and Friedman ([1985]) pointed out, drawing on Newman's critical review (Newman [1928]) of a version of structuralism advocated by Russell ([1927]), the ramsification procedure yields a claim which is, roughly speaking, equivalent to the claim that the theory is empirically adequate. Actually, it is more complicated than this, as we show below.

\section{Technical Framework}

To investigate this matter more closely ${ }^{3}$, we shall consider theories formulated in an interpreted, two-sorted, second-order language, denoted $L_{2}\left(\left\langle O_{i}\right\rangle,\left\langle M_{i}\right\rangle,\left\langle T_{i}\right\rangle\right)$. The extra-logical predicates of this language are interpreted by three sequences of realworld relations: $\left\langle O_{i}\right\rangle=\left(O_{1}, O_{2}, \ldots\right)$ (the 'observational' relations), $\left\langle M_{i}\right\rangle=\left(M_{1}, M_{2}\right.$, ...) (the 'mixed' relations) and $\left\langle T_{i}\right\rangle=\left(T_{1}, T_{2}, \ldots\right)$ (the 'theoretical' relations). ${ }^{4}$ For ease of notation, we shall henceforth write ' $O$ ', ' $M$ ', etc. instead of ' $\left\langle O_{i}\right\rangle$ ', ' $\left\langle M_{i}\right\rangle$ ', etc., for

\footnotetext{
${ }^{2}$ Psillos ([1999]) has become a canonical defence of scientific realism. See Sokal and Bricmont ([2001]) for an important discussion of the sense in which mature scientific theories in physics approximate theoretical structure of the world (in particular, what they call the 'Renormalization Group view' of ontology).

${ }^{3}$ The technical framework described here — an interpreted language whose intended semantics is 'carved up' according to the epistemic status (i.e., observability) of the entities and relations it refers to-is riddled with further problems. It is the best analysis I can give of the framework of the antirealist opponent, and the primary aim here is to show that this anti-realist framework (at least, as I understand it) doesn't achieve its intended goal.

${ }^{4}$ The omission of functions (which we use to represent most physical quantities) is no problem, since functions may be reduced to many-one relations in well-known ways. A simpler but defective possibility is to consider a one-sorted second-order language $L_{2}(O, T)$ which contains no mixed relations. A further possibility is to consider a 3-sorted notation, with variable sorts for observable entities (chairs, bar magnets, etc.), theoretical entities (say, space-time points/events, or point particles) and for mathematical entities (say, sets, real numbers, or functions of reals, etc.).
} 
the appropriate sequences of observational, mixed, etc., relations. The first-order variables of this language $L_{2}(O, M, T)$ comprise two-sorts: primary first-order variables (say $x_{1}, x_{2}, \ldots$ ) ranging over observable objects, such as chairs, bar magnets, iron filings, voltmeter pointers, etc.; and secondary first-order variables (say $y_{1}, y_{2}, \ldots$ ) ranging over non-observable objects, such as space-time points, point particles, real numbers, etc. Correspondingly, there are three classes of predicates and predicate variables: primary predicates denoting the observable relations and primary predicate variables $X_{1}, X_{2}, \ldots$, ranging over observable relations (the field of any primary or observable relation is some subset of the class of observable entities); secondary predicates denoting theoretical relations and quantities and secondary predicate variables $Z_{1}, Z_{2}, \ldots$, ranging over theoretical relations and quantities (the field of any secondary or theoretical relation is some subset of the class of non-observable objects); and finally mixed predicates denoting mixed relations and mixed predicate variables $Y_{1}, Y_{2}, \ldots$ ranging over mixed relations, which are relations between observable objects and non-observable ones. ${ }^{5}$ For simplicity, the predicate symbols denoting the relations $O_{i}, M_{i}$ and $T_{i}$ are also called $O_{i}, M_{i}$ and $T_{i}$ (nothing turns on this later on). ${ }^{6}$ The result of deleting both secondary (theoretical) and mixed predicate symbols from the interpreted language $L_{2}(O, M, T)$ yields a still second-order and two-sorted language which we denote $L_{2}(O)$. The result of deleting second-order variables then yields the one-sorted, first-order 'empirical' or observational sublanguage, denoted $L(O)$.

A finitely axiomatized scientific theory expressed in $L_{2}(O, M, T)$ may be represented as a single axiom, say $\Theta(O, M, T)$. A Henkin structure $\mathbf{M}$ for the language $L_{2}(O, M, T)$ has the messy two-sorted form,

$$
\left(\left(D_{1}, D_{2}\right), \operatorname{Rel}, R_{O}, R_{M}, R_{T}\right)
$$

where $D_{1}$ is the primary domain over which observational variables range; $D_{2}$ is the secondary or 'theoretical' domain over which theoretical variables range; the total domain of the model, as it were, is just the union $D_{1} \cup D_{2}$; the Henkin structure also specifies a 'relation domain' Rel, which is some collection of classes and relations on the total domain $D_{1} \cup D_{2}$, satisfying the comprehension scheme and certain other conditions connected to the variable sorts. ${ }^{7}$ If all relations are included in $\mathrm{Rel}$, then the Henkin structure is called full. The (sequences of) relations $R_{O}, R_{M}$ and $R_{T}$ are the specific relations in $\mathbf{M}$ which interpret the corresponding primitive predicates of $L_{2}(O$, $M, T)^{8}$

\footnotetext{
${ }^{5}$ Again, to note another aspect of the oddity of this framework, many scientifically significant relations and quantities (e.g., various space-time relations and quantities, various scientific quantities such as mass, length, duration, location, etc.) will 'decompose' into three strangely distinct relations, depending upon the observational status of their relata. In any case, in order to make sense of ramsification, one is forced into some such distinction.

${ }^{6}$ By analogy, in the model theory of arithmetic, we take $s$ to be both the standard successor operation on the natural numbers, and also to be the symbol in the language of arithmetic that denotes this operation. Given an interpretation $\mathbf{M}$ (possibly non-standard) for the language of arithmetic, the operation that the symbol $s$ denotes is then $s^{\mathbf{M}}$.

${ }^{7}$ To be more precise, further constraints are imposed to respect the restrictions on variable sorts, so that for example an observational relation cannot 'mix' observable and non-observable entities. An observational relation must be a relation on $D_{1}$, and a theoretical relation must be a relation on $D_{2}$. A mixed relation must be a relation on $D_{1} \times D_{2}$. More exactly, a mixed relation is a subset of $\left(D_{1}\right)^{n} \times$ $\left(D_{2}\right)^{m}$.

${ }^{8}$ For further details concerning the apparatus of second-order logic, see Shapiro 1991, van Dalen 1994 or Manzano 1996.
} 
To emphasize, the theory formulation language $L_{2}(O, M, T)$ is interpreted. By 'interpreted' we mean semantically interpreted, rather than epistemologically interpreted (e.g., by Carnap-Bridgman style correspondence rules). The worldly relations that this language is 'hooked up' to are $O_{i}, M_{i}$ and $T_{i}$. Furthermore, we are here working within some informal set-theoretic meta-language, and this meta-theory refers to these relations too. A mistake of positivist philosophy of science was to suppose that one must work within a finitistic or syntactical (say, 'Hilbertian') metatheory. It seems to me still mistaken to adopt solely a fully mathematical meta-theory, with the resources to define certain kinds of mathematical structures, such as Einstein manifolds, fibre bundles, Fock spaces, and so on (as Suppes recommended). If one wishes to talk about truth at all, then the meta-language must be an extension of the scientific object language, and must be a 'Tarskian' meta-language, with the crucial property of containing the interpreted object language expressions ('the electromagnetic field $F_{a b}$,' 'the space-time metric tensor $g_{a b}$, , etc.). If we wish to talk about truth conditions for scientific statements, then we must do this. For example, in modern differential forms terminology, one of the statements of Maxwell's theory is 'The exterior derivative of the electromagnetic field $F$ is zero' (in jargon, $F$ is a closed 2-form): this claim about the world is true just in case the exterior derivative of the electromagnetic field $F$ is indeed zero. Unless one has, at minimum, a translation of object language to the meta-language, then a materially adequate theory of truth for the object language cannot be given: a minimal constraint on a theory of truth is that it satisfies Tarski's Convention T. ${ }^{9}$

The observational domain $D_{O}$ of the intended interpretation is the union of the fields of the observational relations. Similarly, the theoretical domain $D_{T}$ of the intended interpretation is the union of the fields of the theoretical relations. The whole first-order domain of the intended interpretation is the union of the observable domain $D_{O}$ and the theoretical domain $D_{T}$. We also make the assumption that the intended relation domain for the intended model is full, so it contains all classes and polyadic relations (satisfying the afore-mentioned restrictions). Thus we may define the intended (full) structure for $L_{2}(O, M, T)$ as the full structure $\left(\left(D_{O}, D_{T}\right), O, M, T\right)$. In a sense, this is the 'slice' of the world according to this particular language.

Next, we define what it means to say of a sentence of $L_{2}(O, M, T)$ that it is true:

Definition A: $\varphi$ is a true sentence of $L_{2}(O, M, T)$ if and only if $\left(\left(D_{O}, D_{T}\right), O\right.$, $M, T) \vDash \varphi$.

Restricted to the observational sublanguage $L(O)$, the definition of truth is:

Definition B: $\varphi$ is a true sentence of $L(O)$ if and only if $\left(D_{O}, O\right) \vDash \varphi$.

Since we are dealing with arbitrary $L_{2}(O, M, T)$-structures, we can define what it means for such a structure $\mathbf{M}$ to be 'ontologically correct': roughly, we take this to

\footnotetext{
${ }^{9}$ If the meta-language is impoverished, it is clear that the notion of truth is not definable in the metatheory. The truth values of sentences about electrons depend upon the properties of electrons (and not on 'pointer-readings', etc.). For the full statement of Convention T, see Tarski ([1935/6]). It is important not to confuse the semantical notion of truth, with its central disquotational feature, with the semantical relation of a sentence's being true in a model, a notion which Tarski also defined, but in a different paper (Tarski ([1936]). There are subtle questions concerning Tarskian truth definitions which satisfy Convention $\mathrm{T}$ (the material adequacy condition), and Tarski's model-theoretical notion of a sentence's being true when interpreted over a structure.
} 
mean 'isomorphic to the real world'. From the 'point of view' of the interpreted language $L_{2}(O, M, T)$, the world $i$ the intended structure $\left(\left(D_{O}, D_{T}\right), O, M, T\right)$. So,

Definition C: A full $L_{2}(O, M, T)$-structure $\left(\left(D_{1}, D_{2}\right), R_{O}, R_{M}, R_{T}\right)$ is ontologically correct just in case $\left(\left(D_{1}, D_{2}\right), R_{O}, R_{M}, R_{T}\right) \approx\left(\left(D_{O}, D_{T}\right), O, M, T\right)$.

Then we have,

Theorem 1: A set $\Theta$ of sentences in $L_{2}(O, M, T)$ is true if and only if it has an ontologically correct model.

Proof: For the left-to-right direction, suppose $\Theta$ is true. Clearly, it has an ontologically correct model, namely $\left(\left(D_{O}, D_{T}\right), O, M, T\right)$. For the right-to-left direction, suppose that $\Theta$ has an ontologically correct model $\mathbf{M}=\left(\left(D_{1}, D_{2}\right)\right.$, $\left.R_{O}, R_{M}, R_{T}\right)$. Let $\varphi \in \Theta$. Then, $\left(\left(D_{1}, D_{2}\right), R_{O}, R_{M}, R_{T}\right) \vDash \varphi$. Since this model M is isomorphic to the intended model, we have $\left(\left(D_{O}, D_{T}\right), O, M, T\right) \vDash \varphi$. So, $\varphi$ is true. Hence, all sentences in $\Theta$ are true.

For an analogy, if the interpreted language under discussion were $L(\mathbf{N})$, the interpreted language of arithmetic, then the intended interpretation is just the natural number structure $\mathbf{N}$. A structure for this language is 'ontologically correct' just in case it is isomorphic to $\mathbf{N}$. And a theory in this language is true just in case it has a standard model.

\section{Ramsification}

A delicate question concerns the construction of the Ramsey sentence, since our interpreted language refers to 'mixed' or 'bridge' relations (the $M_{i}$ ). I have seen no explicit discussion of this issue. Pending further clarification, we ramsify the predicates denoting the mixed relations also. So, on this assumption, the Ramsey sentence includes a clause to the effect that 'there exist mixed relations from the observable domain to the theoretical domain'. On this understanding, the Ramsey sentence $\mathfrak{R}(\Theta)$ has the second-order form, $\exists Y \exists Z \Theta(O, Y, Z)$ (here ' $Y$ ' and ' $Z$ ' stand for sequences of second-order variables). The Ramsey sentence is a sentence in the language $L_{2}(O)$. This language is still two-sorted. In particular, it contains first-order variables and quantifiers ranging over the domain of non-observable objects. One cannot meaningfully ramsify variables. The Ramsey sentence thus does not eliminate quantification over non-observable objects. It does eliminate predicates which denote specific theoretical relations. So, (full) models for the Ramsey sentence must have the two-sorted form $\left(\left(D_{1}, D_{2}\right), R_{O}\right)$, incorporating some domain $D_{2}$ of 'theoretical' objects (as opposed to relations, quantities, etc).

Intuitively, the Ramsey sentence for a theory $\Theta$ now expresses the following propositional content:

There exist theoretical relations on the theoretical domain $D_{T}$, and there exist mixed relations connecting $D_{T}$ to the observable domain $D_{O}$, such that $\Theta$ holds with respect to these theoretical and mixed relations and the actual observational ones.

In general, the Ramsey sentence $\Re(\Theta)$ is a deductive consequence of the theory $\Theta$, in standard second-order logic. So a Ramsey sentence $\mathfrak{R}(\Theta)$ is no stronger than the original theory $\Theta$. With the apparatus developed thus far, we can show: 
Theorem 2: $\left(\left(D_{1}, D_{2}\right), \operatorname{Rel}, R_{O}\right) \vDash \mathfrak{R}(\Theta)$ if and only if there exists a sequence $R_{M}$ of 'mixed' relations and a sequence $R_{T}$ of 'theoretical' relations in $R e l$ such that $\left(\left(D_{1}, D_{2}\right), R e l, R_{O}, R_{M}, R_{T}\right) \vDash \Theta$.

Proof: Just commute the existential quantifiers in $\mathfrak{R}(\Theta)$ outside the satisfaction relation $\vDash$.

So, a Henkin structure for the language $L_{2}(O)$ satisfies a Ramsey sentence just in case it can expanded in a certain way, adding mixed and theoretical relations, yielding an $L_{2}(O, M, T)$-structure which satisfies $\Theta$.

Theorem 3: The theories $\Theta$ and $\mathfrak{R}(\Theta)$ prove the same $L_{2}(O)$-sentences. In particular, $\Theta$ is a conservative extension of $\Re(\Theta)$ for $L(O)$-sentences.

Proof: $\Theta$ proves all $L_{2}(O)$-sentences that $\mathfrak{R}(\Theta)$ does, since $\Theta$ proves $\mathfrak{R}(\Theta)$. Now, suppose there is an $L_{2}(\mathrm{O})$-sentence $\varphi$ such that $\Theta$ proves $\varphi$ and $\Re(\Theta)$ does not prove $\varphi$. By Henkin's completeness theorem, there exists a (not necessarily full) $L_{2}(O)$-structure $\left(\left(D_{1}, D_{2}\right), \operatorname{Rel}, R_{O}\right) \vDash \mathfrak{R}(\Theta)$ such that $\left(\left(D_{1}\right.\right.$, $\left.\left.D_{2}\right), \operatorname{Rel}, R_{O}\right) \vDash \neg \varphi$. So, there exist 'theoretical' relations $R_{T}$ and 'mixed' relations $R_{M}$ in $\operatorname{Rel}$ such that $\left(\left(D_{1}, D_{2}\right), \operatorname{Rel}, R_{O}, R_{M}, R_{T}\right) \vDash \Theta$. Since $\Theta$ proves $\varphi$, we have that $\left(\left(D_{1}, D_{2}\right), R e l, R_{O}, R_{M}, R_{T}\right) \vDash \varphi$ and since $\varphi$ lacks theoretical predicates, $\left(\left(D_{1}, D_{2}\right), R e l, R_{O}\right) \vDash \varphi$. Contradiction.

So, $\Theta$ and $\Re(\Theta)$ prove the same $L_{2}(O)$-sentences. And note that $L_{2}(O)$ is a powerful language, containing first-order quantification over the domain $D_{T}$ of non-observable objects, and also second-order quantification over relations amongst non-observables. Only when we pass to the one-sorted empirical sub-language language $L(O)$ do we fully renounce quantification over both non-observable objects and relations amongst them.

From the above, we obtain:

Theorem 4: $\mathfrak{R}(\Theta)$ is true if and only if there exists a sequence $R_{T}$ of theoretical relations (on $D_{T}$ ) and mixed relations $R_{M}$ (on $D_{\mathrm{O}} \times D_{T}$ ) such that $\left(\left(D_{O}, D_{T}\right), O, R_{M}, R_{T}\right) \vDash \Theta$.

That is, $\mathfrak{R}(\Theta)$ is true just in case the 'observable fragment' $\left(D_{O}, O\right)$ of the world can be expanded to satisfy the theory $\Theta$.

Let us now consider a toy example of ramsification. Let Tim be the set of time instants and let Before be the 'before' ordering on Tim. A realist might propose the following theory of time, referring specifically to the set Tim and the relation Before:

(1) $\quad($ Tim, Before $) \approx(\mid \mathrm{R},<)$.

This says that 'the physical ordering of time is order-isomorphic to the natural ordering of the continuum'. If the notions of 'time instant' and 'before' are regarded as theoretical, then ramsification of (1) eliminates these references to specific theoretical parts of the world (namely, the set Tim of time instants and the relation Before) and replaces the theory (1) with the following 'structural' assertion,

(2) There exists a subset $T$ of entities in the external world and there exists a binary relation $B$ on $T$ such that $(T, B) \approx(\mid \mathrm{R},<)$. 
Our knowledge claims about time are 'purely structural': we only know that there is some set 'out there' with some relation 'out there' on this set, which satisfies our 'structural description': i.e., is isomorphic to the mathematical structure $(\mid \mathrm{R},<)$.

Discussing the Ramsey sentence construction, several authors have urged that the Ramsey sentence for a theory yields something extremely close to the instrumentalist claim that the theory is empirically adequate. For example, English ([1973]) shows that empirically adequate Ramsey sentences cannot contradict one another. But the most important discussion of this matter given by Demopoulos and Friedman ([1985]), who urge that,

The problem with this procedure [ramsification] is that it trivializes physics: it threatens to turn the empirical claims of science into mere mathematical truths. More precisely, if our theory is consistent, and if all its purely observational consequences are true, then the truth of the Ramsey sentence follows as a theorem of set theory or second-order logic, provided our initial domain has the right cardinality-if it doesn't, then the consistency of our theory again implies the existence of a domain that does. Hence, the truth of physical theory reduces to the truth of its observational consequences. (Demopoulos and Friedman 1985, p. 635).

The technical claim here - call it The DF Claim — rests on arguments given by Newman ([1928]), a review of Russell's Analysis of Matter ([1927]). Newman argued that Russell's 'structuralism' is prone to a serious trivialization argument. This argument rests on a mathematical point, which can be stated as an easy theorem of set theory:

Let $X$ be a set/collection and let $\mathbf{M}$ be a structure such that $|\operatorname{dom}(\mathbf{M})| \leq|X|$. Then there exists a structure $\mathbf{M}_{X}$ whose domain is $X$ with a substructure isomorphic to $\mathbf{M}$.

It is worth sketching the proof for later. Let $\mathbf{M}$ have the form $\left(D, R_{1}, \ldots, R_{k}\right)$. By assumption, $|D| \leq|X|$. So, there is an injection $\phi: D \rightarrow X$. Let $X^{\phi}$ be the range of $\phi$. Clearly, $\phi$ is a bijection from $D$ to $X^{\phi}$ and induces relations $R_{i}^{\phi}$ on $X$ as follows. Suppose $R_{i} \subseteq D^{n}$ is a distinguished $n$-place relation in the structure $\mathbf{M}$. Then define the 'pushed forward' relation $R_{i}^{\phi}$ :

$$
R_{i}^{\phi}={ }_{d f}\left\{\phi(\underline{d}):(\underline{d}) \in R_{i}\right\}
$$

(where $(\underline{d})$ is a sequence of the form $\left(d_{1}, \ldots, d_{n}\right)$ ). Let $\mathbf{M}_{X}$ be the structure $\left(X, R_{1}{ }^{\phi}\right.$, $\left.\ldots, R_{k}^{\phi}\right)$. Then, the structure $\left(X^{\phi}, R_{1}^{\phi}, \ldots, R_{k}^{\phi}\right)$ is a substructure of $\mathbf{M}_{X}$ and is by construction isomorphic to $\mathbf{M}$.

Intuitively put:

Given any set/class $X$, we can impose any structure $\mathbf{M}_{X}$ we like onto $X$ subject only to cardinality constraints.

Returning to Friedman and Demopoulos's application of this, I essentially agree with their conclusion. Ramsification turns the contingent theoretical claims of physics (about theoretical quantities and relations, such as the electromagnetic field, the 
metric structure of space-time, etc.) into 'mere mathematical truths'. This is surely absurd. We might call it physics without physics. ${ }^{10}$

On the other hand, it is important to examine more carefully the crucial technical DF claim here. ${ }^{11}$ The DF claim is that 'the truth of the Ramsey sentence follows as a theorem of set theory or second-order logic', under the condition that the theory is satisfiable and all observational consequences are true. We show below that the DF claim is not quite correct, although it is very close to being correct. The technical subtlety here concerns the formulation of the empirical adequacy claim, to which we now turn.

\section{Empirical Adequacy}

In this section, we spend some time examining formulations of the notion of empirical adequacy. A standard explication of the notion of 'empirical adequacy' is to say that 'all consequences of the theory in the observational sub-language are true'. This leads to a notion we shall call weak empirical adequacy:

Definition D: A theory $\Theta$ in $L_{2}(O, M, T)$ is weakly empirically adequate just in case, for any $L(O)$-sentence $\varphi$, if $\Theta \vdash \varphi$, then $\varphi$ is true.

However, we can express a stronger model-theoretic notion of empirical adequacy, by capturing the following idea, discussed by van Fraassen:

... a theory is empirically adequate exactly if what it says about the observable things and events in this world, is true-exactly if it 'saves the phenomena'. A little more precisely: such a theory has at least one model that all the phenomena fit inside. (van Fraassen [1980], p. 12).

How do we capture this in the present framework? Let us consider possible $L_{2}(O$, $M, T$ )-structures. Intuitively, for any such structure $\mathbf{M}$, we want the empirical reduct of $\mathbf{M}$ to be 'isomorphic to the appearances'-i.e., isomorphic to the empirical or observational part of the real world. Given that the intended structure is $\left(\left(D_{O}, D_{T}\right), O\right.$, $M, T)$, the 'empirical part' of this is just the reduct $\left(D_{O}, O\right)$. So, we say that,

Definition E: A full $L_{2}(O, M, T)$-structure $\left(\left(D_{1}, D_{2}\right), R_{O}, R_{M}, R_{T}\right)$ is empirically correct just in case $\left(D_{1}, R_{O}\right) \approx\left(D_{O}, O\right)$.

Following van Frasssen's suggestion, empirical adequacy of $\Theta$ can be defined in terms of $\Theta$ having an empirically correct full model:

\footnotetext{
${ }^{10}$ There is a strong tendency within structural realism, and structuralism more generally, to replace the theoretical parts of physics with uninterpreted pure mathematics. A similar point can be made in relation to the so-called 'model-theoretic view' of scientific theories. If one identifies a scientific theory $\Theta$ with a collection $\Sigma$ of mathematical structures, then the question arises as to what is meant by saying that some structure $\mathbf{M} \in \Sigma$ represents the physical world. If one independently identifies the 'physical world' with some physical structure $\mathbf{P}$ (thereby 'interpreting' any structure $\mathbf{M}$ in $\Sigma$ ), then one can simply require that $\mathbf{M}$ be isomorphic to $\mathbf{P}$. But this requires a theory-independent interpretation of the mathematics. If the mathematical structures in $\Sigma$ are 'uninterpreted', then to say that $\mathbf{M}$ represents the physical world is to say this: there exist physical relations $R_{1}, \ldots, R_{n}$ on the domain $D_{P}$ of physical things such that $\mathbf{M} \approx\left(D_{P}, R_{1}, \ldots, R_{n}\right)$. This is clearly a ramsified claim and, by Newman reasoning, this claim is true just in case the physical domain $D_{P}$ is sufficiently large.

${ }^{11}$ The DF claim has been disputed by Zahar and Worrall. See the jointly-authored Appendix to Zahar ([2001]).
} 
Definition F: A theory $\Theta$ is empirically adequate just in case it has an empirically correct full model.

It may seem odd to require this, for it permits the possibility that theory $\Theta$ might have another full model which is not empirically correct. However, the oddity is dispelled if we recall that, as we showed above, a theory is true just in case it has an ontologically correct model. Empirical adequacy in this sense is having a model whose empirical part is ontologically correct. Empirical adequacy really is a restricted sort of truth. Furthermore, despite the prima facie difference between the definitions of weak empirical adequacy and empirical adequacy, one can easily prove that empirical adequacy entails weak empirical adequacy:

Theorem 5: If a theory $\Theta$ is empirically adequate, then it is weakly empirically adequate.

Proof: Assume that $\Theta$ is empirically adequate as defined above. So, there exists an empirically correct full model $\mathbf{M}=\left(\left(D_{1}, D_{2}\right), R_{O}, R_{M}, R_{T}\right) \vDash \Theta$ with $\left(D_{1}, R_{O}\right) \approx\left(D_{\mathrm{O}}, O\right)$. Let $\varphi$ be an $L(O)$-sentence which $\Theta$ proves. Since $\left(\left(D_{1}\right.\right.$, $\left.\left.D_{2}\right), R_{O}, R_{M}, R_{T}\right)$ is a model of $\Theta$, we have $\left(\left(D_{1}, D_{2}\right), R_{O}, R_{M}, R_{T}\right) \vDash \varphi$. Since $\varphi$ lacks theoretical vocabulary, we have $\left(D_{1}, R_{O}\right) \vDash \varphi$. Now, $\left(D_{1}, R_{O}\right) \approx\left(D_{\mathrm{O}}, O\right)$. Hence, $\left(D_{O}, O\right) \vDash \varphi$. So, $\varphi$ is true. Since $\varphi$ was arbitrary, we infer that all empirical deductive consequences of $\Theta$ are true. So, $\Theta$ is weakly empirical adequate.

The converse of this theorem does not hold. Indeed, it is possible in principle that there might be genuine counter-examples: theories which are weakly empirically adequate, but not empirically adequate in the sense of possessing a standard model of the empirical part of the world. A real counter-example would presumably depend upon contingent facts about the world and, in particular, facts about whether the domain of observables is infinite.

But an instructive toy example may be given from mathematical logic, where we reinterpret the notion of 'empirical adequacy' so as to apply to arithmetical theories. Consider theories formulated in the uninterpreted language $L_{T r}$, the language of arithmetic extended by a monadic predicate symbol $T r$, which we intend to behave like a truth predicate. We stipulate that the interpretation of the arithmetic part of $L_{T r}$ is the (standard) natural number structure N. Say that a theory formulated in this language is weakly empirically adequate if all of its arithmetic theorems are true in the standard model $\mathbf{N}$. Say that a theory is empirically adequate if it has a model $\mathbf{M}$ isomorphic to $\mathbf{N}$. Now, consider the theory of truth we denote $F S^{*}$ (closely related to a system presented in Friedman and Sheard 1987; see Halbach 1999, pp. 368-9). FS* contains the axioms of Peano arithmetic, extended by certain axioms and rules for the predicate $T r$, intuitively satisfying certain conditions on a truth predicate. It follows from a result due to McGee ([1985]) that $F S^{*}$ is $\omega$-inconsistent. There is a formula $\varphi(x)$ in $L_{T}$, containing the predicate $T r$, such that,

$$
F S^{*} \vdash \neg \varphi(\mathbf{n}) \text {, for each number } n \in \mathbf{N} \text {, }
$$

$$
F S^{*} \vdash \exists x \varphi(x)
$$

It follows that if $(\mathbf{M}, X)$ is a model of $F S^{*}$ (where $X \subseteq \operatorname{dom}(\mathbf{M})$ is a set which interprets the predicate symbol $T r$ ), then the arithmetical reduct $\mathbf{M}$ is non-standard. 
So, in particular, $F S^{*}$ is not 'empirically adequate' in the sense defined above. However, Halbach has shown that $F S^{*}$ is a conservative extension of $P A$ (see Halbach [1999], Theorem 4.2). ${ }^{12}$ Since all arithmetic consequences of $P A$ are true, it follows that all arithmetic consequences of $F S^{*}$ are true. It follows that $F S^{*}$ is 'weakly empirically adequate'.

This shows that a theory may be weakly empirically adequate and yet not empirically adequate. So, weak empirical adequacy, defined in terms of the truth of all observational consequences, is properly weaker than the model-theoretical notion of empirical adequacy, defined in terms of having an empirically correct model.

\section{Ramsification $\approx$ Empirical Adequacy + Cardinality Condition}

In this section we clarify the instrumentalist nature of the Ramsey sentence construction. We ask: what exactly is the relation between the empirical adequacy claim and the Ramsey sentence? The answer is that the truth of the Ramsey sentence for a theory $\Theta$ is mathematically equivalent to the theory's possession of an empirically correct full model $\mathbf{M}$ whose theoretical domain is the same size as that of the real theoretical domain of the interpreted language.

First, we define a condition, amounting to the requirement that the theoretical domain $D_{2}$ of a given structure $\mathbf{M}$ has the same cardinality as the real theoretical domain $D_{T}$ of the intended structure. We say that,

Definition G: A full $L_{2}(O, M, T)$-structure $\left(\left(D_{1}, D_{2}\right), R_{O}, R_{M}, R_{T}\right)$ is Tcardinality correct if $D_{2} \approx D_{T}$.

The main theorem giving the content of the Ramsey sentence is this:

Theorem 6: $\mathfrak{R}(\Theta)$ is true if and only if $\Theta$ has a full model $\mathbf{M}$ which is Tcardinality correct and empirically correct.

Proof: For left-to-right direction, suppose $\mathfrak{R}(\Theta)$ is true. So, there exists a (full) expansion $\left(\left(D_{O}, D_{T}\right), O, R_{M}, R_{T}\right)$ of the reduct $\left(\left(D_{O}, D_{T}\right), O\right)$ of the intended structure, such that $\left(\left(D_{O}, D_{T}\right), O, R_{M}, R_{T}\right)$ satisfies $\Theta$. This full model of $\Theta$ is obviously empirically correct and T-cardinality correct.

For the right-to-left direction, suppose that $\Theta$ has a T-cardinality correct and empirically correct full model, $\mathbf{M}=\left(\left(D_{1}, D_{2}\right), R_{O}, R_{M}, R_{T}\right)$. Because this model is empirically correct, the observational reduct $\left(D_{1}, R_{O}\right)$ is 'isomorphic to the appearances': $\left(D_{1}, R_{O}\right) \approx\left(D_{\mathrm{O}}, O\right)$, given by some bijection $\phi: D_{1} \rightarrow D_{O}$. And because this model is T-cardinality correct, there is another bijection $\psi: D_{2} \rightarrow$ $D_{T}$. We use these bijections $\phi$ and $\psi$ to define a (full) structure $\mathbf{M}=\left(\left(D_{\mathrm{O}}, D_{T}\right)\right.$, $\left.O, R_{M}{ }^{*}, R_{T}^{*}\right)$ and show that this satisfies $\Theta$. We use the isomorphisms $\phi: D_{1}$

\footnotetext{
${ }^{12}$ Note that the system $F S^{*}$ does not expand the Induction Scheme of $P A$ to formulas containing the truth predicate $\operatorname{Tr}$. (This is what the * indicates). Full $F S$ is the result of expanding induction to include all formulas. Axiomatic theories of truth over $P A$ which $d o$ expand the Induction Scheme are sometimes non-conservative extensions (for example, the theory of truth based on the Tarskian inductive clauses). In a sense, the truth axioms lead to new (and indeed true) theorems about the purely non-semantical (i.e., arithmetic) domain. For example, one can prove the Global Reflection Principle for the non-semantical theory. This leads to an interesting argument against deflationism about truth. See Shapiro ([1998]) and Ketland ([1999]) for formulations of this argument.
} 
$\rightarrow D_{O}$ and $\psi: D_{2} \rightarrow D_{T}$ to define new mixed relations $\left(R_{M}\right)_{i}^{*}$, and new theoretical relations $\left(R_{T}\right)_{i}{ }^{*}$ as follows:

$$
\begin{aligned}
& \left(R_{M}\right)_{i}^{*}=_{\mathrm{df}}\left\{(\phi(\underline{x}), \psi(\underline{y})):(\underline{x}, \underline{y}) \in\left(R_{M}\right)_{i}\right\} \\
& \left(R_{T}\right)_{i}{ }^{*}=_{\mathrm{df}}\left\{\psi(\underline{y}):(\underline{y}) \in\left(R_{T}\right)_{i}\right\}
\end{aligned}
$$

(where $\underline{x}$ and $y$ are sequences of appropriate lengths). We have now combined $\phi$ and $\psi$ to an 2-sorted isomorphism $(\phi, \psi)$ :

$$
(\phi, \psi):\left(\left(D_{1}, D_{2}\right), R_{O}, R_{M}, R_{T}\right) \rightarrow\left(\left(D_{\mathrm{O}}, D_{T}\right), O, R_{M}^{*}, R_{T}^{*}\right) .
$$

Hence, $\left(\left(D_{\mathrm{O}}, D_{T}\right), O, R_{M}^{*}, R_{T}^{*}\right) \vDash \Theta$. Hence, $\left(\left(D_{O}, D_{T}\right), O\right) \vDash \mathfrak{R}(\Theta)$. So, $\Re(\Theta)$ is true.

\section{Conclusion}

Let us return to the DF claim, that '... if our theory is consistent, and if all its purely observational consequences are true, then the truth of the Ramsey sentence follows as a theorem of set theory or second-order logic' (Demopoulos and Friedman [1985], p. 635). This corresponds in our terminology to the claim that if $\Theta$ is satisfiable and weakly empirically adequate, then $\mathfrak{R}(\Theta)$ is true. And this is not quite the case, because weak empirical adequacy is properly weaker than empirical adequacy, as the $F S^{*}$ example shows. However, the DF claim is almost correct. For we have shown that the truth of the Ramsey sentence is equivalent to a sort of combination of empirical adequacy and a Newman-esque domain cardinality constraint. Indeed, the 'structural content' of a theory $\Theta$, at least if it is identified with what $\mathfrak{R}(\Theta)$ 'adds' to the claim that $\Theta$ is empirically adequate, is just this Newman-esque cardinality constraint. This leaves the structural realist in a sticky position, given that the epistemological intention was to provide an interesting third way between anti-realism and realism: the position collapses to something very close to anti-realism. As Friedman and Demopoulos put it: ramsification 'trivializes physics: it threatens to turn the empirical claims of science into mere mathematical truths'.

Faculty of Philosophy

University of Cambridge

Cambridge CB3 9DA

jjk32@cam.ac.uk

\section{Acknowledgements}

I would like to thank attendees of the Structural Realism conference held at LSE in March 2002 (particularly Ioannis Votsis for organizing the meeting); also members of informal HPS seminar at Leeds; and three anonymous referees. 
Carnap, R. [1956]: The Philosophical Foundations of Physics, edited by M. Gardner. New York: Basic Books. Reprinted as An Introduction to the Philosophy of Science, New York: Dover Books, 1995.

Demopoulos, W and M. Friedman [1985]: 'Critical Notice: Bertrand Russell's The Analysis of Matter: Its Historical Context and Contemporary Interest', Philosophy of Science, 52, pp. 621-39.

English, J. [1973]: 'Underdetermination: Craig and Ramsey', Journal of Philosophy, 70, pp. 453-62.

Friedman, H. and Sheard, M. [1987]: 'An Axiomatic Approach to Self-Referential Truth', Annals of Pure and Applied Logic, 33, pp. 1-21.

Halbach, V. [1999]: 'Conservative Theories of Classical Truth', Studia Logica 62, pp. 353-70.

Ketland, J. [1999]: 'Deflationism and Tarski's Paradise', Mind 108, pp. 69-94.

Manzano, M. [1996]: Extensions of First-Order Logic, Cambridge Tracts in Theoretical Computer Science, Vol. 19, Cambridge: Cambridge University Press.

McGee, V. [1985]: 'How Truthlike can a Predicate Be? A Negative Result', Journal of Philosophical Logic, 14, pp. 399-411.

Newman, M.H.A. [1928]: 'Mr Russell's Causal Theory of Perception', Mind, 37, pp. 137-48.

Psillos, S. [1999]: Scientific Realism: How Science Tracks Truth. London: Routledge.

Ramsey, F.P. [1929]: 'Theories', in R.B. Braithwaite (ed.), 1931, Foundations of Mathematics, London: Routledge and Kegan Paul.

Russell, B.A.W [1927]: Analysis of Matter, London: Allen and Unwin.

Shapiro, S. [1991]: Foundations without Foundationalism: A Case for Second-Order Logic, Oxford: Oxford University Press.

Shapiro, S. [1998]: 'Truth and Proof - Through Thick and Thin', Journal of Philosophy 95, pp. 493-521.

Sokal, A and J. Bricmont [2001]: 'Defence of a Modest Scientific Realism', Talk given at Bielefeld-ZIF Conference on 'Welt und Wissen - Monde et Savoir - World and Knowledge', $18^{\text {th }}$ June 2001.

Tarski, A. [1935/6]: 'Der Wahrheitsbegriff in den formalisierten Sprachen', Studia Philosophica 1: 261-405. Translation by J.H. Woodger 'The Concept of Truth in Formalized Languages', in A. Tarski 1956, Logic, Semantics and Metamathematics. Oxford: Clarendon Press.

Tarski, A. [1936]: 'Über den Begriff der logischen Folgerung', Actes du Congrès Internationale de Philosophie Scientifique Vol. 7, pp. 1-11. Translation by J.H. Woodger 'On The Concept of Logical Consequence', in A. Tarski 1956, Logic, Semantics and Metamathematics, Oxford: Clarendon Press.

van Dalen, D. [1994]: Logic and Structure, Berlin: Springer.

van Fraassen, B.C. [1980]: The Scientific Image, Oxford: Clarendon Press. 
Worrall, J. [1989]: 'Structural Realism: The Best of Both Worlds?', Dialectica, 43, pp. 99-124. Reprinted in Papineau (ed.) 1996, Philosophy of Science, Oxford: Oxford University Press.

Zahar, E. [2001]: Poincaré's Philosophy: From Conventionalism to Phenomenology. Chicago: Open Court. 